\title{
Gastroesophageal reflux in patients with idiopathic pulmonary fibrosis referred for lung transplantation
}

\author{
Matthew P. Sweet, MD, ${ }^{a}$ Marco G. Patti, MD, ${ }^{a}$ Lorriana E. Leard, MD, ${ }^{b}$ Jeffrey A. Golden, MD, ${ }^{b}$ Steven R. Hays, MD, \\ Charles Hoopes, MD, and Pierre R. Theodore, MD ${ }^{a}$
}

From the University of California San Francisco, Departments of Surgery a and Medicine, ${ }^{\mathrm{b}}$ San Francisco, Calif.

Read at the Eighty-sixth Annual Meeting of The American Association for Thoracic Surgery, Philadelphia, Pa, April 29-May 3, 2006.

Received for publication May 8, 2006; revisions received Aug 7, 2006; accepted for publication Sept 5, 2006.

Address for reprints: Pierre R. Theodore, MD, 350 Parnassus, Suite 150, San Francisco, CA 94143 (E-mail: theodorep@ surgery.ucsf. edu).

J Thorac Cardiovasc Surg 2007;133:1078-84 $0022-5223 / \$ 32.00$

Copyright $\odot 2007$ by The American Association for Thoracic Surgery

doi:10.1016/j.jtcvs.2006.09.085
Objectives: The association between gastroesophageal reflux disease and idiopathic pulmonary fibrosis has not been fully characterized. The aims of this study were to determine in patients with idiopathic pulmonary fibrosis (1) the prevalence of reflux symptoms, (2) the esophageal manometric profile, and (3) the prevalence of proximal and distal esophageal reflux.

Methods: Between May 1999 and March 2006, 30 patients with idiopathic pulmonary fibrosis were referred to the Swallowing Center at the University of California San Francisco. Each patient underwent a structured symptom assessment, esophageal manometry, and 24-hour dual sensor ambulatory $\mathrm{pH}$ monitoring.

Results: Twenty (67\%) patients had abnormal esophageal reflux. Typical reflux symptoms, although more common in those with reflux, were not reliable as a screening test (sensitivity $65 \%$, specificity $71 \%$ ). Sixty-five percent of patients with abnormal reflux had a hypotensive lower esophageal sphincter. Abnormal esophageal peristalsis was more common among those with reflux (50\% vs $10 \% ; P=.03)$. In $9(30 \%)$ patients, acid refluxed into the proximal esophagus for over $1 \%$ of the study time.

Conclusions: A majority of patients with idiopathic pulmonary fibrosis have pathologic reflux. Symptoms do not distinguish between those with and without reflux. In these patients, reflux is associated with a hypotensive lower esophageal sphincter and abnormal esophageal peristalsis, and often extends into the proximal esophagus.

$\mathrm{I}$ diopathic pulmonary fibrosis (IPF) is a lethal form of interstitial lung disease that affects 30,000 to 50,000 people in the United States. The pathophysiology appears to involve aberrant fibroblast proliferation owing to recurrent epithelial injury. ${ }^{1}$ The etiology is unknown. Currently, there is no effective medical therapy for IPF, and median survival is only 3 years.

Several lines of evidence suggest an association between gastroesophageal reflux disease (GERD) and lung fibrosis. Thirty years ago, Mays, Dubois, and Hamilton ${ }^{2}$ found that 48 patients with lung fibrosis of unknown etiology had twice the prevalence of hiatal hernia and GERD as compared with patients with lung fibrosis of known etiology. Patients with erosive esophagitis had a 1.36 odds ratio of pulmonary fibrosis in a case control study of more than 200,000 US veterans. ${ }^{3}$ More recently, studies of patients undergoing lung transplantation have found that reflux is common among patients with end-stage lung disease. ${ }^{4,5}$ Furthermore, distal esophageal reflux and markers of microaspiration have been associated with the early onset of chronic allograft failure after transplantation, suggesting that GERDrelated microaspiration causes direct lung injury. 5,6

In the absence of an identifiable etiology, speculation has arisen that reflux may play a role in the pathogenesis or progression of IPF. ${ }^{7}$ Reflux is of particular interest because it represents a treatable process. Recently, a case series demonstrated that 


\section{Abbreviations and Acronyms \\ ATS/ERS = American Thoracic Society/European Respiratory Society \\ DLCO = diffusion of carbon monoxide in the lung \\ $\mathrm{FEV}_{1}=$ forced expiratory volume in 1 second \\ GERD = gastroesophageal reflux disease \\ IPF = idiopathic pulmonary fibrosis \\ LES = lower esophageal sphincter \\ UCSF = University of California San Francisco \\ UES = upper esophageal sphincter}

patients with end-stage IPF undergoing antireflux surgery had reduced supplemental oxygen dependence, demonstrating the safety and potential efficacy of antireflux surgery in these patients. ${ }^{8}$

At this time, little is known about the prevalence or etiology of reflux in patients with IPF, and there is no accepted standard for screening. This retrospective study of patients with IPF referred for lung transplantation was done to determine (1) the prevalence of distal and proximal reflux, (2) the esophageal manometric profile, and (3) whether or not reflux symptoms could be used to screen for reflux.

\section{Patients and Methods}

One hundred twenty patients referred for lung transplantation underwent esophageal testing in the University of California San Francisco (UCSF) Swallowing Center between 1999 and March 2006. Of these, 36 had a diagnosis of IPF. Six subjects were studied after lung transplantation, leaving a total of 30 patients who completed evaluation before transplantation. This study focuses on this cohort of 30 pretransplant patients with IPF. Sixteen of these patients were included in an earlier report. ${ }^{9}$ A report describing the esophageal testing of 109 patients with all causes of lung disease referred for transplantation has been published elsewhere. ${ }^{10}$ Referral for esophageal testing was routine for all pretransplant patients since July 2003. Twenty-six subjects were evaluated after that time.

The pathologic diagnosis of usual interstitial pneumonia was established by surgical lung biopsy or by pathologic review of the explanted lung in 25 subjects. The remaining 5 subjects were given a diagnosis of IPF on the basis of the American Thoracic Society/ European Respiratory Society (ATS/ERS) consensus statement criteria. ${ }^{11}$ Patients with nonspecific interstitial pneumonia were excluded.

Clinic charts and electronic medical records were reviewed for all patients. The best estimate of steroid use and time interval between diagnosis of IPF and date of esophageal studies was extracted from these records. These results were not prospectively confirmed during evaluation in the Swallowing Center. Endoscopic examination of the upper esophagus was performed only when clinically indicated.

\section{Symptomatic Evaluation}

Each subject underwent a standardized interview with the Swallowing Center physician or technician. Patients estimated the se- verity of their symptoms (heartburn, regurgitation, dysphagia) with a 5-point scale ranging from 0 (no symptoms) to 4 (disabling symptoms). They were also questioned about the use of acidreducing medications (histamine 2-blocking agents and proton pump inhibitors).

\section{Esophageal Manometry}

Esophageal manometry was performed after an overnight fast by techniques previously described. ${ }^{12}$ Medications that might interfere with esophageal motor function (ie, calcium-channel blocking agents, nitrates, and metoclopramide) were discontinued at least 48 hours before the study. Position, pressure, length, and relaxation of the lower esophageal sphincter (LES) were measured by the station pull-through technique, with $0.5-\mathrm{cm}$ increments between stations. Esophageal body function was recorded 3, 8, 13, and $18 \mathrm{~cm}$ above the upper border of the LES by giving 10 swallows of $5 \mathrm{~mL}$ of water at 30-second intervals. Amplitude of peristaltic waves was calculated independently for the distal ( 3 and $8 \mathrm{~cm}$ above the LES) and the proximal esophagus (13 and $18 \mathrm{~cm}$ above the LES). Esophageal length was measured from the upper esophageal sphincter (UES) to the distal end of the LES. Ineffective esophageal motility was defined as distal esophageal amplitude less than $30 \mathrm{~mm} \mathrm{Hg}$ or greater than $30 \%$ simultaneous waves in the distal esophagus. ${ }^{13}$ Nonspecific esophageal motility disorder was defined as the presence of abnormal esophageal peristalsis not meeting the criteria for ineffective esophageal motility.

\section{Ambulatory pH Monitoring}

Proton pump inhibitors were withheld for 14 days and histamine-2 blockers were withheld for 3 days before the study in all patients. The $\mathrm{pH}$ catheters were calibrated in a standard buffer solution at $\mathrm{pH} 1$ and $\mathrm{pH} 7$ before and after monitoring. A dual-channel $\mathrm{pH}$ catheter with two antimony sensors located $15 \mathrm{~cm}$ apart was placed transnasally, with the distal sensor $5 \mathrm{~cm}$ above the upper border of the manometrically determined LES. Patients were instructed to consume an unrestricted diet, refrain from taking any acid-suppressing medications, and to keep a diary of symptoms during the $\mathrm{pH}$ testing. Data were incorporated into a composite score (DeMeester score). A score greater than 14.7 was set as abnormal on the basis of data obtained from 50 volunteers. ${ }^{14}$ The cohort was divided into two groups, GERD positive or negative, on the basis of the DeMeester score. Proximal reflux was defined as greater than $1 \%$ total time that $\mathrm{pH}$ was less than 4 at the proximal sensor. ${ }^{15}$ The data were analyzed with a commercial software program (Gastrosoft; Medtronic Functional Diagnostic, Shoreview, Minn).

\section{Statistical Analysis}

Statistical analysis was performed with STATA Statistical Software: Release 9.1 (Stata Corporation, College Station, Tex). Nonparametric statistical analyses were performed. Differences between the two groups were analyzed with the $\chi^{2}$ test for proportions or the Mann-Whitney test for continuous variables. Data are reported as number (percentage) from $\chi^{2}$ analysis or as median (interquartile range) for nonparametric tests. Spearman rank correlation was used to examine associations between continuous or ordinal variables. 
TABLE 1. Demographics and pulmonary function tests

\begin{tabular}{lccl}
\hline & GERD + & GERD - & $P$ value \\
\hline Demographics & & & \\
No. of patients & 20 & 10 & \\
Age & $62(56-64)$ & $58(54-61)$ & .3 \\
Male & $17(85 \%)$ & $7(70 \%)$ & .33 \\
BMI & $27(25-29)$ & $27(25-30)$ & .51 \\
Steroid use & $12(80 \%)$ & $8(53 \%)$ & .12 \\
PPI use before & $14(70 \%)$ & $5(50 \%)$ & .28 \\
$\quad$ study & & & \\
Pulmonary function & & & \\
FEV $1 \%$ predicted) & $76(65-94)$ & $53(52-58)$ & .0004 \\
DLCO (\% predicted) & $3962(2157-6873)$ & $2153(1240-3257)$ & .000627
\end{tabular}

$B M I$, Body mass index; $P P I$, proton pump inhibitor; $F E V_{1}$, forced expiratory volume in 1 second; $D L C O$, diffusion of carbon monoxide in the lung. Continuous data are presented as the number and (percentage). Categorical data are presented as the median and (interquartile range).

The study protocol was approved by the UCSF Committee for Human Subjects Research.

\section{Results}

Thirty patients completed their evaluation in the Swallowing Center at UCSF. The patients with and without reflux did not differ with respect to age, gender, body mass index, or use of proton pump inhibitors before esophageal evaluation (Table 1). Median estimated time between IPF diagnosis and esophageal testing was 22 months (range 1-121 months). A majority of subjects had been prescribed proton pump inhibitors empirically or in conjunction with steroid administration for management of IPF. Fifteen (50\%) patients were receiving steroid therapy at the time of referral to the Swallowing Center. Abnormal reflux was seen in $80 \%$ of patient not receiving steroids and in $53 \%$ of patients receiving steroids $(P=.12)$ The groups were different with respect to their pulmonary function testing. The group with reflux had significantly better measures of forced expiratory volume in 1 second $\left(\mathrm{FEV}_{1}\right)$ and carbon monoxide diffusion in the lung (DLCO). FEV 1 and DLCO were correlated with DeMeester score ( $r=0.56$ and $0.65, P=.0025$ and .0002$)$.

Five patients had endoscopic examination of the upper esophagus. Hiatal hernias were identified in all 5. Abnormal mucosal changes of the distal esophagus (irregular Z-line and/or erythema) were noted in 3 patients. All 5 subjects had abnormal DeMeester scores and 2 had abnormal proximal reflux.

\section{Symptomatic Assessment}

Twenty-seven subjects completed the symptom evaluation. Sixty-five percent of the subjects with abnormal esophageal acid exposure reported one or more typical symptom, leaving $35 \%$ with asymptomatic reflux. Conversely, among the subjects with normal esophageal acid exposure, $29 \%$ reported typical reflux symptoms (Table 2).
TABLE 2. Prevalence of reflux symptoms

\begin{tabular}{lccc}
\hline & GERD + & GERD - & $\boldsymbol{P}$ value \\
\hline Any typical reflux symptom & $65 \%$ & $29 \%$ & .10 \\
Heartburn & $58 \%$ & $29 \%$ & .19 \\
Regurgitation & $56 \%$ & $14 \%$ & .06 \\
Dysphagia & $39 \%$ & $14 \%$ & .24 \\
\hline
\end{tabular}

To determine the utility of typical reflux symptoms as a screening test for reflux, we compared the report of any one or more typical symptoms with the esophageal $\mathrm{pH}$ findings. Patients who reported experiencing any heartburn, regurgitation, and/or dysphagia were considered to have a positive symptom screening test. These data were then compared in separate $2 \times 2$ tables with the presence or absence of abnormal distal and proximal acid exposure on 24-hour $\mathrm{pH}$ monitoring (Table 3). The sensitivity and specificity for distal reflux were $65 \%$ and $71 \%$, respectively.

\section{Esophageal Manometry}

Patients with reflux had lower median LES pressures (12 vs $20 \mathrm{~mm} \mathrm{Hg} ; P=.01)$ and were more likely to have a hypotensive LES (mean resting pressure $<14 \mathrm{~mm} \mathrm{Hg}$ ) (65\% vs $10 \% ; P=.004$; Table 4 ). Abnormal esophageal peristalsis was more common among patients with reflux (50\% vs $10 \% ; P=.03$; Table 5). In this group, 6 patients had nonspecific esophageal motility disorder $(30 \%)$ and 4 patients had ineffective esophageal motility (20\%). A single patient with a normal distal and proximal esophageal acid exposure had ineffective esophageal motility. Distal esophageal wave duration was significantly shorter in those with reflux, and there was a trend toward weaker distal esophageal wave amplitudes, but this result was not statistically significant. Median UES pressures were lower in those with reflux than in those without (55 vs $84 ; P=.054$ ).

\section{Twenty-four-hour pH Monitoring}

Abnormal distal esophageal acid exposure was found in $67 \%$ of the cohort, and abnormal proximal esophageal acid exposure was found in $30 \%$ (Table 6). The data demonstrate the substantial difference in the amount of reflux between

\section{TABLE 3. Utility of symptomatic screening}

\begin{tabular}{lcc}
\hline & Distal reflux & Proximal reflux \\
\hline Sensitivity & $65 \%$ & $60 \%$ \\
Specificity & $71 \%$ & $39 \%$ \\
Positive predictive value & $87 \%$ & $21 \%$ \\
Negative predictive value & $42 \%$ & $58 \%$ \\
\hline
\end{tabular}

Comparison of the presence or absence of any typical reflux symptoms with esophageal $\mathrm{pH}$ findings. See text. Distal reflux, DeMeester score $>14.7$. Proximal reflux, Proximal probe $\mathrm{pH}<4$ for $>1 \%$ of study time. 
TABLE 4. Esophageal sphincter function

\begin{tabular}{lccl}
\hline & GERD + & GERD - & $P$ value \\
\hline LES pressure $(\mathrm{mm} \mathrm{Hg})$ & $12(9-18)$ & $20(16-24)$ & .01 \\
Hypotensive LES $(<14 \mathrm{~mm} \mathrm{Hg})$ & $13(65 \%)$ & $1(10 \%)$ & .004 \\
Total LES length (cm) & $2.3(1.9-4)$ & $2.9(2.3-3)$ & .51 \\
Abdominal LES length (cm) & $2(1.1-2.3)$ & $2.5(2-3)$ & .21 \\
UES pressure (mm Hg) & $55(27-70)$ & $84(70-106)$ & .054
\end{tabular}

LES, Lower esophageal sphincter; UES, upper esophageal sphincter. Continuous data are presented as the median and (the interquartile range). Categorical data are presented as the number and (percentage).

those with and without reflux. Mean esophageal acid clearance time was longer among patients with reflux.

Proximal reflux was found in 9 (30\%) patients. The majority of proximal reflux occurred in the upright position. One patient with normal distal acid exposure had abnormal proximal esophageal acid exposure. This patient has normal esophageal motility and a normal LES.

In addition to the 30 patients who were studied before transplantation, 6 additional patients with pathologic diagnoses of IPF were studied after lung transplantation. All 6 patients were found to have elevated DeMeester scores (median 45, range 38-83) and 3 were found to have abnormal proximal acid exposure.

\section{Discussion}

These data demonstrate that (1) reflux was prevalent and frequently extended into the proximal esophagus, (2) reflux was associated with a hypotensive LES and abnormal esophageal peristalsis, and (3) symptoms were not a reliable means of screening patients with IPF for reflux.

Symptoms have been shown not to be predictive of respiratory symptoms attributed to GERD. ${ }^{16-18}$ This appears to hold true among patients with IPF. Raghu and colleagues ${ }^{19}$ reported that typical symptoms were found in $47 \%$ of 65 patients with IPF. D'Ovidio and colleagues ${ }^{4}$

TABLE 5. Esophageal body function

\begin{tabular}{lccc}
\hline & GERD + & GERD - & $P$ value \\
\hline Normal peristalsis & $10(50 \%)$ & $9(90 \%)$ & .03 \\
NSEMD & $6(30 \%)$ & 0 & .05 \\
IEM & $4(20 \%)$ & $1(10 \%)$ & .49 \\
DEA (mm Hg) & $78(49-116)$ & $109(77-145)$ & .10 \\
DED (mm Hg) & $3(2.8-3.7)$ & $4.3(4-5)$ & .01 \\
PEA (mm Hg) & $45(31-87)$ & $61(54-67)$ & .34 \\
PED (mm Hg) & $2.3(2-2.8)$ & $3(2.7-3.2)$ & .12 \\
Esophageal length (cm) & $27(24-28)$ & $26(25-30)$ & .70 \\
\hline
\end{tabular}

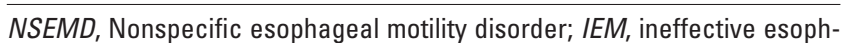
ageal motility; $D E A$, distal esophageal amplitude; $D E D$, distal esophageal duration; $P E A$, proximal esophageal amplitude, $P E D$, proximal esophageal duration. Continuous data are presented as the median and (the interquartile range). Categorical data are presented as the number and (percentage).
TABLE 6. Esophageal pH findings

\begin{tabular}{lccc}
\hline & GERD + & GERD - & $P$ value \\
\hline Distal probe & & & \\
No. of reflux events & $213(129-280)$ & $36(27-57)$ & $<.00005$ \\
Events $>5$ min & $7(4-10)$ & $0(0-1)$ & .0001 \\
Percent time pH $<4$ & $15(9-21)$ & $1.3(0.6-2)$ & $<.00005$ \\
DeMeester score & $52(38-85)$ & $6(4-8)$ & $<.00005$ \\
Proximal probe & & & \\
No. of reflux events & $21(10-68)$ & $4(2-7)$ & .012 \\
Events $>5$ min & $0(0-1)$ & 0 & .06 \\
Percent time pH $<4$ & $0.7(0.1-2.4)$ & $0(0-0.2)$ & .23 \\
Percent time upright & $1(0.2-3)$ & $0(0-0.4)$ & .01 \\
Percent time supine & $0.2(0-1)$ & 0 & .025 \\
Proximal reflux & $8(40 \%)$ & $1(10 \%)$ & .09 \\
Clearance time & $1(0.8-1.2)$ & $0.4(0.3-0.7)$ & 0.0014 \\
Distal clearance & $0.3(0.1-0.7)$ & $0(0-0.3)$ & 0.06 \\
Proximal clearance & & \\
\hline
\end{tabular}

Continuous data are presented as the median and (the interquartile range). Categorical data are presented as the number and (percentage). Distal reflux is defined as DeMeester score $>14.7$. Proximal reflux is defined as $\mathrm{pH}<4$ at the proximal probe for $>1 \%$ of the study time. Mean clearance times are calculated by dividing the number of minutes $\mathrm{pH}<4$ and dividing by the number of reflux episodes. Units are minutes/episode.

reported that $65 \%$ of 26 subjects with all-cause interstitial lung disease referred for transplantation reported having reflux symptoms. Neither of these studies reported the different prevalence of symptoms among those with and without GERD. Our results demonstrate that symptoms cannot be used to screen patients with moderate-to-severe IPF for reflux. Objective esophageal testing is the only means currently available to screen for reflux in these patients.

Esophageal manometry demonstrated that reflux was associated with a hypotensive LES. Furthermore, 50\% of those with reflux had abnormal esophageal peristalsis. In these patients, the two physiologic antireflux mechanisms are impaired. The LES, the main barrier to reflux, is weak. Furthermore, the esophageal peristalsis is abnormal, so that the refluxate remains in the esophagus for a longer period of time. This combination results in prolonged esophageal exposure to reflux, which we suspect increases the likelihood of aspiration. ${ }^{13}$

The prevalence of distal and proximal reflux was $67 \%$ and $30 \%$, respectively. Raghu and colleagues ${ }^{19}$ found a prevalence of distal and proximal reflux of $76 \%$ and $63 \%$ among 46 patients with IPF. In that study, different proximal esophageal testing procedures were used, with the proximal probe at $15 \mathrm{~cm}$ above the LES or $2 \mathrm{~cm}$ above the UES. The difference in measured prevalence of proximal reflux could be due to these methodological differences, chance, or to referral bias. Proximal reflux is of particular importance inasmuch as it has been associated with respiratory symptoms attributed to reflux and microaspiration. ${ }^{16,20}$ Among the 6 patients who were studied after transplantation, distal 
and proximal reflux were seen in $100 \%$ and $50 \%$, respectively. Because transplant has been shown to worsen reflux, these patients were not included in the main analysis of this study. ${ }^{21}$

One patient had normal distal acid exposure but abnormal proximal acid exposure. Larger studies of patients with all-cause end-stage lung disease have also identified a subset of patients with a similar reflux profile. ${ }^{4}$ In these patients, the quantity and frequency of distal reflux is physiologic, but most of that reflux extends into the proximal esophagus. Single-channel distal esophageal $\mathrm{pH}$ monitoring, therefore, will fail to identify this subgroup of patients at increased risk for aspiration.

In our cohort, the patients with reflux had better pulmonary function testing as measured by both $\mathrm{FEV}_{1}$ and DLCO. This finding was contrary to our expectations. Raghu and coworkers ${ }^{19}$ found no correlation between pulmonary function testing and amount of reflux. In that study, the 6 patients without reflux had lower mean values for forced vital capacity and DLCO. Statistical analysis was limited by the small group size. We are suspicious that the association between pulmonary function and reflux is a result of chance, despite the strong statistical significance of the rank-sum test. Alternatively, it may reflect an unidentified referral bias. Increased negative intrathoracic inspiratory pressures and increased positive intra-abdominal pressures during cough may contribute to reflux in patients with lung disease. Surprisingly, in this study, patients with less severe lung disease had more reflux, suggesting that an increased thoracoabdominal pressure gradient is not the sole cause of reflux.

The principal limitations of this study are the small sample size and the potential for referral bias, inasmuch as the patients were seen in the context of referral for lung transplantation. Therefore, caution must be used when generalizing these findings to a broader population of patients with IPF.

Esophageal $\mathrm{pH}$ monitoring underestimates the amount and frequency of reflux. Unlike esophageal impedence testing, $\mathrm{pH}$ monitoring does not detect nonacidic reflux or measure the volume of the refluxate. Future studies should use impedance technology.

In conclusion, this study shows that $30 \%$ of patients with IPF have gastroesophageal reflux that extends into the proximal esophagus, putting them at high risk for recurrent microaspiration. Whether or not such reflux plays a causal role in IPF pathogenesis, progression, or acute exacerbation is unknown. Ultimately, reflux is a surrogate marker of aspiration, and clarification of the role of reflux in IPF will depend on demonstrating that reflux and/or aspiration are risk factors for disease progression or death. These data support further efforts to study this association. Ultimately, if such an association is causal, antireflux surgery may be therapeutic for a subgroup of patients with IPF, blocking the progression of disease and perhaps avoiding the need for transplantation.

\section{References}

1. Harari S, Caminati A. Idiopathic pulmonary fibrosis. Allergy. 2005; 60:421-35

2. Mays EE, Dubois JJ, Hamilton GB. Pulmonary fibrosis associated with tracheobronchial aspiration. A study of the frequency of hiatal hernia and gastroesophageal reflux in interstitial pulmonary fibrosis of obscure etiology. Chest. 1976; 69:512-5.

3. El-Serag H, Sonnenberg A. Comorbid occurrence of laryngeal or pulmonary disease with esophagitis in United States military veterans. Gastroenterology. 1997; 113:755-60.

4. D'Ovidio F, Singer LG, Hadjiliadis D, Pierre A, Waddell TK, de Perrot $\mathrm{M}$, et al. Prevalence of gastroesophageal reflux in end-stage lung disease candidates for lung transplant. Ann Thorac Surg. 2005;80:1254-60.

5. Cantu E 3rd, Appel JZ 3rd, Hartwig MG, Woreta H, Green C, Messier R, et al. J. Maxwell Chamberlain Memorial Paper. Early fundoplication prevents chronic allograft dysfunction in patients with gastroesophageal reflux disease. Ann Thorac Surg. 2004;78:1142-51; discussion 1142-51.

6. D'Ovidio F, Mura M, Tsang M, Waddell TK, Hutcheon MA, Singer LG, et al. Bile acid aspiration and the development of bronchiolitis obliterans after lung transplantation. J Thorac Cardiovasc Surg. 2005 129:1144-52.

7. Raghu G. The role of gastroesophageal reflux in idiopathic pulmonary fibrosis. Am J Med. 2003;115(Suppl 3A):60S-4S.

8. Linden PA, Gilbert RJ, Yeap BY, Boyle K, Deykin A, Jaklitsch MT, et al. Laparoscopic fundoplication in patients with end-stage lung disease awaiting transplantation. J Thorac Cardiovasc Surg. 2006;131:438-46.

9. Patti MG, Tedesco P, Golden J, Hays S, Hoopes C, Meneghetti A, et al. Idiopathic pulmonary fibrosis: how often is it really idiopathic? J Gastrointest Surg. 2005;9:1053-8.

10. Sweet MP, Herbella FAM, Leard L, Hoopes C, Golden J, Hays S, et al. The prevalence of distal and proximal gastroesophageal reflux in patients awaiting lung transplantation. Ann Surg. 2006;244:491-7.

11. American Thoracic Society. American Thoracic Society/European Respiratory Society International Multidisciplinary Consensus Classification of the Idiopathic Interstitial Pneumonias. Am J Crit Care Med. 2002;165:277-304

12. Patti MG, Fisichella P, Perretta S. Preoperative evaluation of patients with gastroesophageal reflux disease. J Laparoendoscop Adv Surg Tech. 2001;11:327-31.

13. Diener U, Patti MG, Molena D, Fisichella PM, Way LW. Esophageal dysmotility and gastroesophageal reflux disease. J Gastrointest Surg. 2001;5:260-5.

14. Jamieson JR, Stein HJ, DeMeester TR. Ambulatory pH monitoring: normal values, optimal thresholds, specificity, sensitivity and reproducibility. Am J Gastroenterol. 1992;87:1102-11.

15. Dobhan R, Castell DO. Normal and abnormal proximal esophageal acid exposure: results of ambulatory dual-probe $\mathrm{pH}$ monitoring. Am $J$ Gastroenterol. 1993;88:25-9.

16. Patti MG, Debas HT, Pellegrini CA. Clinical and functional characterization of high gastroesophageal reflux. Am J Surg. 1993;165:163-8.

17. Oelschlager BK, Chang L, Pope CE 2nd, Pellegrini CA. Typical GERD symptoms and esophageal $\mathrm{pH}$ monitoring are not enough to diagnose pharyngeal reflux. J Surg Res. 2005;128:55-60.

18. Tomonaga T, Awad ZT, Filipi CJ, Hinder RA, Selima M, Tercero F Jr, et al. Symptom predictability of reflux-induced respiratory disease. Dig Dis Sci. 2002;47:9-14.

19. Raghu G, Freudenberger TD, Yang S, Curtis JR, Spada C, Hayes J, et al. High prevalence of abnormal acid gastro-oesophageal reflux in idiopathic pulmonary fibrosis. Eur Respir J. 2006;27:136-42.

20. Farrell S, McMaster C, Gibson D, Shields MD, McCallion WA. Pepsin in bronchoalveolar lavage fluid: a specific and sensitive method of diagnosing gastroesophageal reflux-related pulmonary aspiration. J Pediatr Surg. 2006;41:289-93.

21. Young LR, Hadjiliadis D, Davis RD, Palmer SM. Lung transplantation exacerbates gastroesophageal reflux disease. Chest. 2003;124:1689-93.

22. Martinez FJ, Safrin S, Weycker D, Starko KM, Bradford WZ, King TE Jr, et al. IPF Study Group. The clinical course of patients with idiopathic pulmonary fibrosis. Ann Intern Med. 2005;142:963-7.

23. Young LR, Hadjiliadis D, Davis RD, Palmer SM. Lung transplantation exacerbates gastroesophageal reflux disease. Chest. 2003;124:1689-93. 


\section{Discussion}

Dr Raphael Bueno (Boston, Mass). As mentioned, GERD has been recently implicated in the etiology of rejection after lung transplantation. This finding has led many of us to explore the incidence of GERD in patients who are candidates for lung transplantation, as was described in this presentation. This study shows a high incidence of documented GERD in patients with IPF referred for transplantation at one center. Two thirds of the patients were found to have reflux, and almost half of those had proximal reflux. Also, many of these patients did not have symptoms. These results are certainly consistent with other reports and should lead to the referral of potential recipients for lung transplantations for routine evaluation for reflux.

The two questions I have, having read the manuscript, are as follows: Does this cohort include all of your patients with IPF referred for lung transplantation? A good control of the mechanism of reflux would be evaluating the non-IPF patients. Have you looked at those patients and what have you found?

Dr Sweet. Thank you very much, Dr Bueno.

This represents almost all of our patients with IPF, but not every single one. There were several patients who were not referred for evaluation, probably about 4 or 5 , and in another 2 or 3 patients the studies were not complete and so they are not included in the analysis here.

In response to your other question, we have looked at 109 of our pretransplant patients thus far, inclusive, obviously, of all causes of end-stage lung disease, and we have found similar prevalences of reflux, both proximal and distal, across the cohorts. This includes patients with pulmonary hypertension and chronic obstructive pulmonary disease, groups in which reflux has not really been considered to play a role in the etiology of the lung disease. We have looked across the board, and it is really quite prevalent.

Dr Mark B. Orringer (Ann Arbor, Mich). There are a number of issues that I would like to have you address just for the sake of clarity. This patient population is often using bronchodilators, which relax smooth muscle and therefore relax LES tone. They also use steroids, which increase gastric acid production. Have you correlated the drugs that the patients in your two groups were taking to see whether there was a causal relationship between what you have determined to be reflux and nonreflux in the two groups?

Dr Sweet. We looked at the use of proton pump inhibitors. I did not look specifically at the number of patients who were receiving steroids or bronchodilators at the time that they were referred for evaluation at the Swallow Center. Seventy percent of the patients with reflux had been started on proton pump inhibitors empirically before referral to the Swallowing Center and 50\% of the patients without reflux had also been started empirically, and most of those were in conjunction with treatment with steroids, but I do not know exactly how many had been receiving steroids at the time of referral.

Dr Orringer. So one issue is whether or not the reflux that we are treating is iatrogenic and not inherent in these patients.

The second issue has to do with interpreting the results of 24-hour $\mathrm{pH}$ monitoring in these patients. When patients with a chronic cough or other similar respiratory symptoms are sent to us for an antireflux operation, having several hundred episodes of "reflux" recorded on 24-hour distal esophageal $\mathrm{pH}$ monitoring, are we to conclude that they are really in desperate straits and need the surgery? I always look at these data with a somewhat jaundiced eye, because so many of these patients are chronic air swallowers and are belching all the time. When you see what is recorded as "reflux episodes," you may actually be looking at documentation of physiologic reflux with belching and not necessarily pathologic reflux. I would like to ask Toni Lerut to comment on this. I do not think that pathologic reflux typically presents with 100 to 200 "reflux episodes" on 24-hour $\mathrm{pH}$ monitoring.

Did you differentiate upright reflux from supine reflux? Many "upright refluxers" in my experience tend to be more physiologic refluxers with frequent belching. At issue is how we definitively prove that aspiration of gastric contents into the tracheobronchial tree is occurring. We heard some time ago about the Bilitech catheter (Medtronic, Inc, Minneapolis, Minn) being used to detect bile reflux. I have not heard much more about this technology recently. What really is needed is a probe that can be passed down the bronchoscope to detect bile in the airways, documenting aspiration of gastric contents. I wonder whether you could comment on this as well.

Dr Sweet. To answer your first question, we did look at upright and supine reflux, and the predominance of reflux was in the upright position. Also, as I tried to point out during the talk, nobody really knows which parameter is the most predictive of risk for microaspiration. We do not know objectively which risk factor is the most dangerous, but a statistically significant higher percentage of the patients have these episodes lasting longer than 5 minutes, and many of them have proximal episodes lasting longer than 5 minutes, that is, 4 or 5 episodes lasting longer than 5 minutes during the 24-hour study period. Someone who has a total of half an hour throughout the course of the day of proximal esophageal acid exposure is at very high risk for microaspiration. However, I understand your point that there is not necessarily a single parameter that we can use to determine who is microaspirating. That gets to your second point, which is that really we are using reflux as a surrogate marker of microaspiration. That is what our group is actually working on. We have started recruiting patients already, looking at markers of microaspiration, specifically pepsin, in both bronchoalveolar lavage samples, and we are also trying to look for a noninvasive way to screen for that. That would provide a much more powerful diagnostic measure to look for what we are concerned about, which is the microaspiration event. Therefore, I concur and we are starting to do that now. I would certainly appreciate hearing the thoughts of anybody else who is working on that.

Dr Antoon E. M. R. Lerut (Leuven, Belgium). In follow-up of Mark Orringer's remarks, I have a comment and not a question.

I think impedance is probably going to give us far better insight into the problems of belching, nonacidic reflux, or perhaps acid coming up into the esophagus with air belching. Clearly, impedance will certainly become a helpful tool to investigate problems of supraesophageal reflux.

Dr Tomasz Grodzki (Szczecin, Poland). I have two questions. First, you mentioned that the patients were in end-stage IPF on the basis of radiologic or biopsy confirmation. While I noticed in one of the slides that the $\mathrm{FEV}_{1}$ was $76 \%$ and DLCO more than $60 \%$, were they really suitable candidates for transplantation? Second, 
did you perform any manometry or $\mathrm{pH}$ measurements postoperatively?

Dr Sweet. To answer your first question, you are correct. I should not have used the word "end-stage." The patients were a mix of subjects with moderate to end-stage IPF. That is certainly one of the limitations of our study; we have kind of a cohort of patients who were referred for evaluation for transplantation. A natural history paper, written by Dr Martinez, ${ }^{22}$ came out of the InterMune study of interferon. This paper showed that nearly $20 \%$ of the patients died within $1 \frac{1 / 2}{2}$ years of follow-up, and more than half of those died of abrupt clinical deterioration without demonstrable progression of their IPF in the time period. Because of that, the pulmonologists in our group are trying to refer the patients for transplantation sooner than they had in the past. That is the reason we have a heterogeneous group of patients with IPF with respect to the degree of their lung disease. I hope that answers your question. It is one of the problems. The only patients we have been able to get into the Swallowing Center are the patients who come through the transplant clinic. The other pulmonologists have not yet gotten on board with this, and this is one of the reasons we did the study - to try to get a broader group of patients with IPF referred.

Concerning the second question, looking at reflux and manometry in the posttransplant setting, we have studied 8 to 10 patients, and I actually do not know what lung disease subgroups they all are. There are at least 6 who have IPF. All 6 with IPF who were studied after transplant had abnormal DeMeester scores. I think the median was in the 40 to 50 range, and half of them had abnormal proximal reflux. So the numbers were higher. Again, there is a paper from Duke ${ }^{23}$ comparing the pretransplant and posttransplant esophageal studies, and these investigators found an increase in the overall prevalence of reflux after transplantation. Our small amount of data corroborates that.

Dr Shaf Keshavjee (Toronto, Ontario, Canada). We have been studying this in detail, as you know, because of the association between reflux and bronchiolitis obliterans after transplantation. As Dr Orringer pointed out, it is likely a combination of effects of the drugs these patients are receiving, the change in the anatomy of their chest as the lungs either contract or expand depending on their lung disease, possible injury to the vagus nerve at the time of transplant, and then the posttransplant medications, such as cyclosporine. We have started to try to tease out the contributions of these various factors.

With respect to the functional issues, we have documented a high prevalence, as you know from our published work, of reflux in the pretransplant patients, which was not greatly increased after transplantation. The surgeons were thankful to see that. But one of the things that needs further study is the supine versus upright issue and, second, confirming it with measurement of bile acids in bronchoalveolar lavage. There is an assay, which is quite accurate, that correlates highly with the development of bronchiolitis obliterans. I think that it is real in these patients. What is also interesting is that a lot of patients did not have acid reflux, but possibly had nonacid reflux or bile reflux. The impedance catheter will more clearly detect these patients. On the other hand, we have also noted that some patients actually experienced an improvement in the degree of reflux after transplantation. We speculated that we had changed where the LES complex sat in the chest relative to the abdomen with the realigning of the anatomy of the chest with a transplant. There is indeed a lot of very interesting work to be done to figure all of this out.

Dr Joshua R. Sonett (New York, NY). I have one question. You are talking about IPF in general. Did you look at nonspecific interstitial pneumonia versus unusual interstitial pneumonia, because at least $40 \%$ to $60 \%$ of our patients with IPF have scleroderma and other mixed connective tissue diseases and a really unfunctional esophagus in a number of ways.

Dr Sweet. We actually excluded all patients with nonspecific interstitial pneumonia. As I said, we had tissue diagnoses in 25 of the 30 patients, and in the other 5 the diagnosis was based on the clinical criteria from the ATS/ERS consensus statement. As I think you are aware, that is not $100 \%$ sensitive and specific, but we did exclude the patients with nonspecific interstitial pneumonia and tried to get as pure an IPF cohort as we could for this study.

Dr Sonett. Unusual interstitial pneumonia is probably a better way of expressing that.

Dr Sweet. Yes, I should say unusual interstitial pneumonia. Thank you.

Dr Keshavjee, your paper on bronchiolitis obliterans syndrome and bile acids has been for me the most stimulating paper on this topic. Concerning your comment, from the general reflux data, we understand about $60 \%$ of patients have duodenal gastroesophageal reflux, so that is where we are going to see the bile. I am optimistic that looking at pepsin, which is a more specific marker for gastroesophageal reflux, will, I hope, identify all those patients who are microaspirating. After we have had a chance to collect some data, it will be interesting to look at which patients have duodenal gastroesophageal reflux and which have just gastroesophageal reflux.

Dr William A. Baumgartner (Baltimore, $M d$ ). I am intrigued by this. This is a little bit different take on Dr Orringer's question. Are there any data that suggest that this causes IPF?

Dr Sweet. I am aware of no data correlating the presence of reflux with outcome and IPF or in terms of seeing patients who have reflux who later have IPF develop. I think that is a very difficult problem to address. Obviously, reflux is highly prevalent. Then how do you monitor those patients long enough to see whether their risk of developing IPF is different from that of the general population? I hope that answers your question.

Dr Baumgartner. Yes. I just wonder whether it is the chicken and the egg here, whether anybody really knows. We know it has been shown that perhaps IPF contributes to bronchiolitis obliterans syndrome, but is it the other way around possibly?

Dr Sweet. I think the only other data that are available are anecdotal. I have spoken briefly with Dr Bueno about this, and I do not want to speak on his behalf. However, I know that in our center we have 1 or 2 patients who underwent antireflux surgery with a diagnosis of IPF and were able then to come off the transplant list. I understand that Dr Bueno has had a similar experience at his institution. I think he said that he had 3 patients. Thus there is anecdotal evidence that patients with IPF undergoing antireflux surgery had a significant clinical benefit. However, it is really hard to generalize from anecdotal data. When we can look both in the pre-antireflux and post-antireflux surgery setting at markers of microaspiration and see that we have stopped that and then those patients who no longer have markers of microaspiration are doing better, that would be the best data that we will be able to generate to support the application of antireflux surgery in these patients. 